\title{
Tracionamento ortodôntico de canino permanente superior: relato de caso clínico
}

\author{
Orthodontic traction of upper permanent canine: clinical case report
}

Tracción ortodóncica de canino superior permanente: reporte de caso clínico

Diego dos Santos Dias ${ }^{1 *}$, Maria Fernanda Carvalho Lopes Silva ${ }^{1}$, Lorena Miranda Lima ${ }^{1}$, Luciano Pereira Pego ${ }^{1}$, Teago José Tomaz de Aquino ${ }^{1}$, Márcia Maria de Araújo을 Natália dos Santos Dias², Karla Dias Castro Dias ${ }^{3}$, Claudiana Donato Bauman ${ }^{4}$, José Mansano Bauman ${ }^{1,4}$.

\section{RESUMO}

Objetivo: Descrever uma abordagem conservadora para tratamento de canino permanente superior impactado, através de tracionamento ortodôntico. Detalhamento do caso: Paciente BJFA, 16 anos, sexo feminino, acompanhada na clínica de Ortodontia da Associação Brasileira de Odontologia (ABO) em Montes Claros-MG, queixou-se de dentes tortos. Ao exame clínico intrabucal verificou-se atresia maxilar, desvio de linha média dentária superior, apinhamento dentário anteroinferior, incisivo lateral superior direito conóide, retenção prolongada de canino decíduo superior direito, cujo sucessor permanente não se apresentava palpável. Os exames de imagem, sobretudo a tomografia computadorizada, confirmaram a presença de canino permanente superior direito impactado, localizado em região palatina. Os caninos permanentes, elementos fundamentais ao equilíbrio funcional e estético do sistema estomatognático, representam a segunda maior frequência de impactação. Podem resultar em complicações como: migração de dentes adjacentes, reabsorções radiculares, cistos dentígeros, perda do comprimento do arco, maior susceptibilidade a infecções e dor referida. O tratamento foi realizado através de aparelho ortodôntico fixo, aplicando a terapêutica de expansão transversal da arcada superior com quadrihélice de Ricketts e tracionamento ortodôntico pela técnica de colagem de acessório associada a cantilever. Considerações finais: As técnicas utilizadas permitiram uma abordagem conservadora e menos dispendiosa, mostrando resultados funcionais e estéticos positivos.

Palavras-chave: Ortodontia, Tração, Dente canino, Dente impactado.

\begin{abstract}
Objective: To describe a conservative approach to treatment of impacted permanent maxillary canine, through orthodontic traction. Case report: Patient BJFA, 16 years old, female, treated at Associação Brasileira de Odontologia (ABO) Orthodontics clinic in Montes Claros-MG, complained of crooked teeth. Clinical examination revealed maxillary atresia, midline deviation in the upper dental arch, anteroinferior dental crowding, conoidal right upper lateral incisor, prolonged retention of right upper deciduous canine, whose permanent successor was not palpable. Imaging exams, especially computed tomography, confirmed the presence of impacted right upper permanent canine, located in the palate. The permanent canines are very important for functional and aesthetic balance in the stomatognathic system. These teeth represent the second higher frequency of impaction. They may result in complications such as: migration of adjacent teeth, root resorption, dentigerous cysts, loss of arch length, increased susceptibility to infections, and pain referred. The treatment was performed through a fixed orthodontic appliance, applying the superior arch transverse expansion therapy with Ricketts' Quad-Helix and orthodontic traction by attachment bonding technique associated with cantilever. Final considerations: The techniques used allowed a conservative and less expensive approach, showing positive functional and aesthetic results.
\end{abstract}

Key words: Orthodontics, Traction, Canine tooth, Impacted tooth.

\footnotetext{
${ }^{1}$ Associação Brasileira de Odontologia (ABO), Montes Claros-MG. * E-mail: diego.caetite@hotmail.com

2 Centro Universitário UniFG (UniFG), Guanambi-BA.

3 Universidade José do Rosário Vellano (UNIFENAS), Belo Horizonte-MG.

${ }^{4}$ Universidade Estadual de Montes Claros (UNIMONTES), Montes Claros-MG.
} 


\section{RESUMEN}

Objetivo: Describir un enfoque conservador para el tratamiento del canino superior permanente impactado por medio de la tracción ortodóncica. Descripción del caso: Paciente BJFA, 16 años, del sexo femenino, tratada en la clínica de Ortodoncia de Associação Brasileira de Odontologia (ABO) en Montes Claros-MG, se quejó de dientes torcidos. El examen clínico reveló atresia maxilar, desviación de la línea media en el arco dental superior, apiñamiento dental anteroinferior, incisivo lateral superior derecho conoidal, retención prolongada del canino deciduo superior derecho, cuyo sucesor permanente no era palpable. Los exámenes de imagen, especialmente la tomografía computarizada, confirmaron la presencia de un canino permanente superior derecho impactado en el paladar. Los caninos permanentes, elementos claves para el equilibrio funcional y estético del sistema estomatognático, representan la segunda frecuencia más alta de impactación. Estos dientes pueden provocar complicaciones como: migración de dientes adyacentes, reabsorción de raíces, quiste dentígero, perdida de la longitud del arco, mayor susceptibilidad a infecciones e dolor referido. El tratamiento se realizó a través de un aparato de ortodoncia fijo, aplicando la terapia de expansión transversal del arco superior con aparato Quad Hélix de Ricketts y la tracción ortodóncica mediante la técnica de unión de accesorio asociada con el cantilever. Consideraciones finales: Las técnicas utilizadas permitieron un enfoque conservador y menos costoso, mostrando resultados funcionales y estéticos positivos.

Palabras clave: Ortodoncia, Tracción, Diente canino, Diente impactado.

\section{INTRODUÇÃO}

A erupção dentária é caracterizada pela trajetória dos dentes no interior dos ossos maxilares, em direção ao plano funcional na cavidade bucal (ALMEIDA RR, et al., 2001; MARCELINO VCS, et al., 2017). Trata-se de um processo fisiológico, no qual os elementos dentários normalmente desenvolvem uma sequência favorável de erupção (CAPPELLETTE M, et al., 2008; CARVALHO AAB, et al., 2017). No entanto, os dentes podem se apresentar impactados, retidos por razões multifatoriais como: hereditariedade, retenção prolongada do canino decíduo e presença de cistos, tumores ou supranumerários na região (PRIMO BT, et al., 2011; ALVES EP, et al., 2014; GEBERT TJ, et al., 2014; SOARES-SANTOS KS, et al., 2018). Tal situação acomete rotineiramente os caninos permanentes, grupo dentário que representa a segunda maior frequência de impactação. Esses elementos podem se encontrar retidos principalmente em região palatina, sendo mais comum no sexo feminino (BISHARA, SE, 1992; DAMANTE SC, et al., 2017; CRUVINEL MOB, et al., 2018).

A erupção ectópica e impactação de caninos permanentes podem se apresentar em até $23,5 \%$ de amostras selecionadas previamente para tratamento ortodôntico (CARDOSO MA, et al., 2011; CAPELOZZA FILHO L, et al., 2011). Essas ocorrências merecem atenção, tendo em vista que os caninos são essenciais ao equilíbrio funcional e estético do sistema estomatognático (DAMANTE SC, et al., 2017). Associadamente, podem manifestar complicações como: migração de dentes adjacentes, reabsorções radiculares, cistos dentígeros, perda do comprimento do arco, maior susceptibilidade à infecção e dor referida (BARIANI RC, 2017; SILVA AC, et al., 2017; DAMANTE SC, et al., 2017, SOARES-SANTOS KS, et al., 2018).

O diagnóstico precoce da impactação é fundamental, evitando tratamentos ortodônticos mais avançados e custosos (CARDOSO MA, et al., 2011; NASCIMENTO LRXC, et al., 2016). A identificação dessa condição e a adoção de conduta apropriada exigem competência do clínico geral e dos especialistas em odontopediatria, cirurgia bucomaxilofacial, periodontia e ortodontia, além de requerer importante colaboração do paciente (CARDOSO MA, et al., 2011).

Os principais sinais clínicos dos caninos superiores não irrompidos são: retenção prologada do canino decíduo ou atraso na erupção do canino permanente, angulação distal da coroa do incisivo lateral superior, ausência de proeminência vestibular na região de pilar canino ou presença de proeminência na região palatina (CRUVINEL MOB, et al., 2018). Os caninos inclusos são localizados principalmente por inspeção visual, palpação e exames radiográficos. Os exames de imagem mais comumente utilizados são: radiografia panorâmica, periapical, oclusal, telerradiografia e tomografia computadorizada de feixe cônico (CARVALHO AAB, et al., 2017). 
O prognóstico merece ser considerado com reservas, tendo em vista diversas limitações do tratamento e múltiplas variáveis envolvidas. Tais detalhes devem ser esclarecidos aos pacientes e responsáveis, a fim de se evitar falsas expectativas (CARDOSO MA, et al., 2011). Dentre as alternativas terapêuticas destacam-se o transplante autógeno, exodontia do canino decíduo, exodontia do canino impactado, exposição cirúrgica e tracionamento ortodôntico, ou ausência de tratamento imediato, exigindo acompanhamento em longo prazo (CARDOSO MA, et al., 2011; MARCELINO VCS, et al., 2017; MACENO IS, et al., 2018). O presente estudo visa descrever uma abordagem conservadora para tratamento de canino permanente superior impactado, através de tracionamento ortodôntico. Esta pesquisa foi conduzida em observância aos princípios éticos, sendo aprovada pelo Comitê de Ética em Pesquisa da Universidade Estadual de Montes Claros (UNIMONTES), conforme parecer consubstanciado ํㅜ 3.650.696.

\section{DETALHAMENTO DO CASO}

Paciente BJFA, leucoderma, 16 anos, sexo feminino, acompanhada por seu responsável, compareceu à clínica de Ortodontia da Associação Brasileira de Odontologia ( $A B O$ ) em Montes Claros-MG apresentando queixa de dentes tortos. A anamnese não evidenciou problemas de saúde geral e hábitos bucais deletérios, concomitantemente, a paciente informou não ter se submetido a tratamento ortodôntico prévio. Ao exame clínico facial frontal, identificou-se característica mesocefálica, simetria facial e presença de selamento labial normal. Em norma lateral, verificou-se um perfil levemente convexo, boa projeção do osso zigomático, ausência de protuberância do osso nasal, ângulo nasolabial e linha queixo-pescoço normais. $A$ análise facial revelou a classificação Padrão I de Capelozza (CAPELOZZA FILHO L, 2012).

Ao exame clínico intrabucal verificou-se atresia maxilar, desvio de linha média dentária superior, apinhamento dentário anteroinferior, presença de incisivo lateral superior direito conóide, dente 12, e relação molar Classe I de Angle (ANGLE EH, 1899). Observou-se a retenção prolongada de canino decíduo superior direito, dente 53 , com ausência de mobilidade. O sucessor, canino superior permanente superior direito, não se apresentava palpável. Os aspectos clínicos foram registrados por modelos de estudo, fotografias extrabucais e fotografias intrabucais iniciais (Figura 1).

Figura 1 - Fotografias intrabucais iniciais
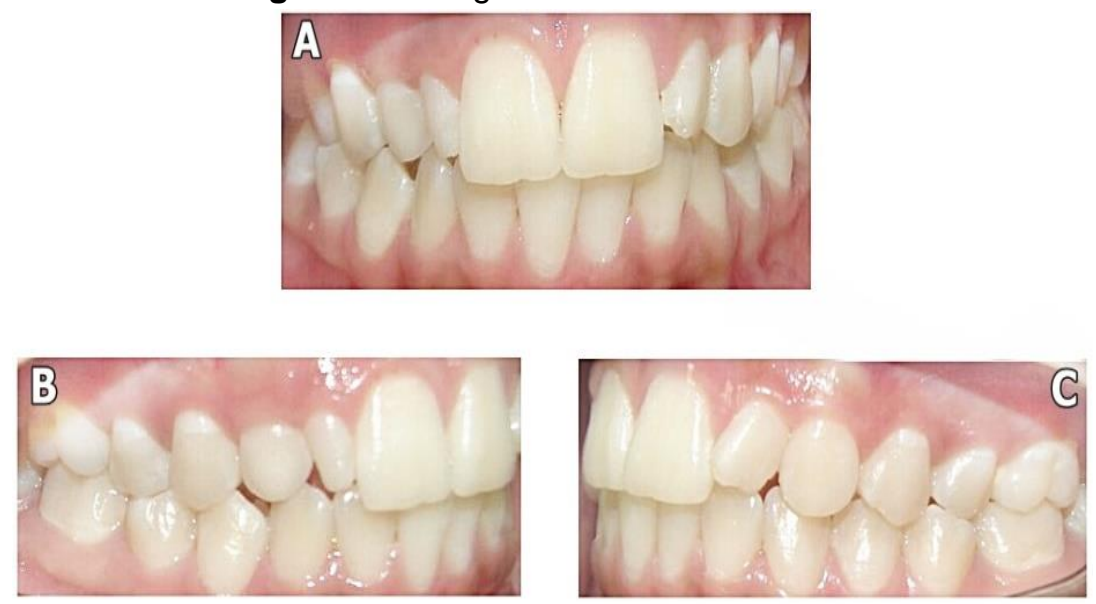

Legenda: A: frontal. B: lateral direita. C: lateral esquerda.

Fonte: Dias DS, et al., 2019.

Realizou-se a avaliação de exames de imagem iniciais: radiografia panorâmica, telerradiografia lateral, radiografias periapicais e tomografia computadorizada da região de canino superior direito. Tais exames, especialmente a tomografia computadorizada, apresentaram concordância com os aspectos clínicos e confirmaram a presença de canino permanente superior direito impactado, dente 13.

A localização na região palatina enquadrou-se na categoria mesioangular da classificação de Winter modificada: longo eixo do canino em posição medial em relação ao longo eixo do incisivo lateral; 
apresentando leve reabsorção radicular, envolvimento até metade da espessura da dentina, conforme a classificação de Ericson que caracteriza as reabsorções de acordo com o grau de comprometimento da estrutura radicular (STIVAROS N e MANDALL NA, 2000; ERICSON S e KUROL PJ, 2000) (Figura 2).

Figura 2 - Exames de imagem iniciais, região de canino permanente superior direito.
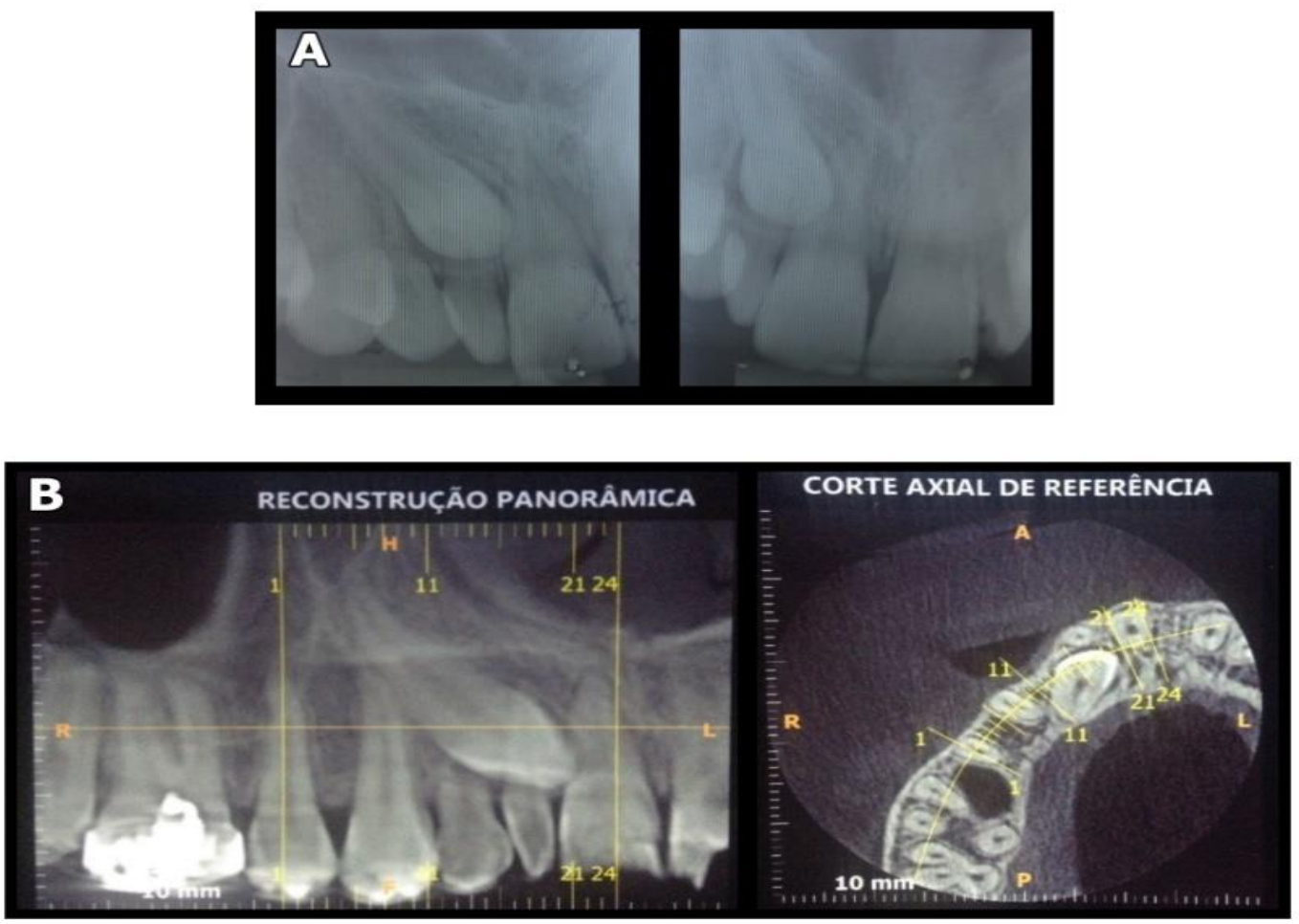

Legenda: A: radiografias periapicais. B: tomografia computadorizada.

Fonte: Dias DS, et al., 2019.

O diagnóstico foi complementado pela análise visual e morfológica dos modelos de estudo e análise cefalométrica, cujas medidas se encontravam dentro dos limites de normalidade. O planejamento ortodôntico objetivou expansão da arcada dentária superior, alinhamento e nivelamento dentário, tracionamento do dente 13, correção da linha média dentária superior e obtenção de espaço para restauração estética do dente 12 .

Primeiramente, iniciou-se a expansão da arcada dentária superior com aparelho quadrihélice de Ricketts (DUARTE MS, 2006). Em seguida, instalou-se aparelho fixo superior, dentes 16 ao 26, e inferior, dentes 46 ao 36, utilizando braquetes prescrição Roth e arcos de níquel-titânio para alinhamento e nivelamento dentário. Após quatro meses de tratamento, obteve-se a dimensão transversal adequada para 0 arco superior e removeu-se o aparelho quadrihélice.

A paciente foi submetida à extração do dente 53 e exposição cirúrgica do dente 13 , sendo realizada colagem de acessório, botão palatino, na coroa canino permanente. Simultaneamente, instalou-se um cantilever no tubo palatino do dente 16 , confeccionado com fio de aço 0.8 , para tracionamento do dente 13 . Foram adicionados helicoides no cantilever para obtenção de vetor extrusivo. Realizou-se ancoragem dos dentes 16,15 e 14 com fio de aço $0.019 \times 0.025$ e amarrilho 0.25 conjugado.

O cantilever foi ativado mensalmente ao dente 13 com amarrilho 0.25 , exercendo leve força de extrusão. Completados quatro meses de tracionamento, removeu-se o cantilever, botão palatino do canino permanente e fio de ancoragem. Realizou-se colagem de braquete no dente 13, promovendo vestibularização, através de leve ativação com amarrilho 0.20 ao arco superior. Assim que o dente 13 atingiu adequada posição, utilizou-se sequência de arcos de aço diagramados e coordenados. 
Respectivamente, foram utilizados elásticos intermaxilares 3/16" médio para intercuspidação dentária. Em seguida, com molas abertas de níquel-titânio, corrigiu-se o desvio da linha média dentária superior e reestabeleceu-se espaço para restauração estética do dente 12. Para conclusão, foi desenvolvido controle de torque em arcos de aço retangulares $0.019 \times 0.025$. A sequência clínica foi representada por meio de fotografias intrabucais oclusais e laterais (Figuras 3 e 4).

Figura 3 - Fotografias oclusais da maxila.
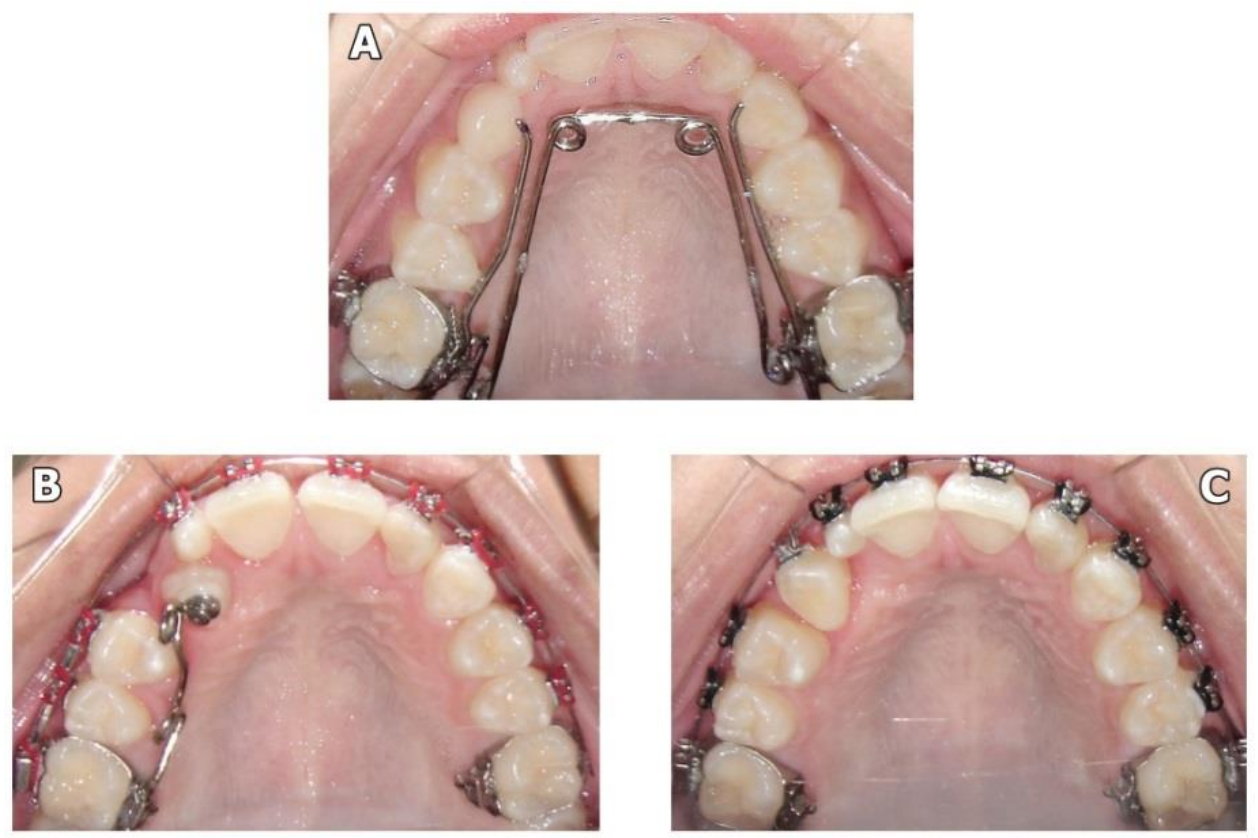

Legenda: A: expansão transversal, aparelho quadrihélice de Ricketts. B: tracionamento do dente 13, técnica de colagem de acessório e uso de cantilever. C: vestibularização do dente 13.

Fonte: Dias DS, et al., 2019.

Figura 4 - Fotografias intrabucais, região lateral direita.
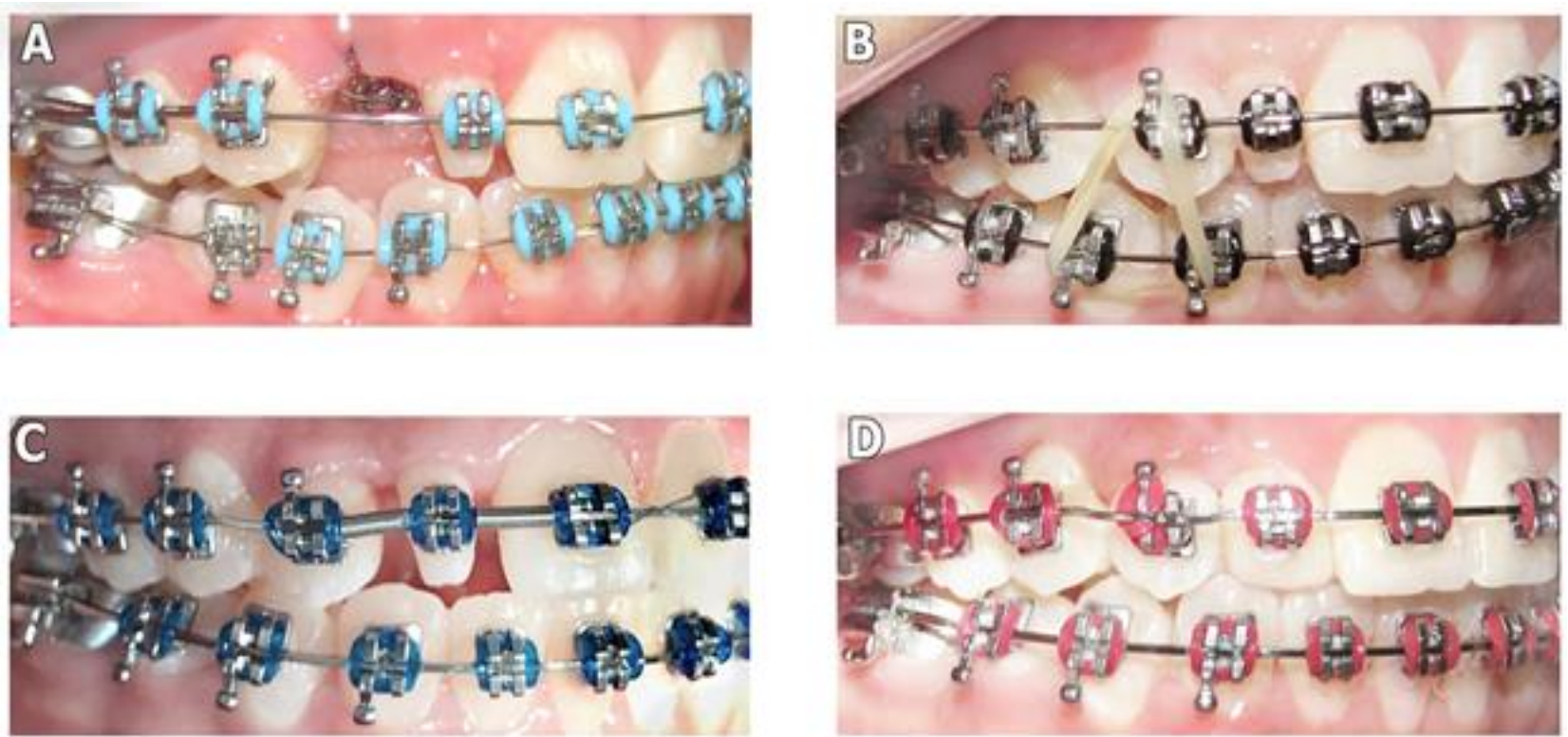

Legenda: A: ancoragem posterior, após extração do dente 53. B: Elásticointermaxilar,após tracionamento do dente 13. C: Obtenção de espaço para restauração estética do dente 12. D: Controle de torque.

Fonte: Dias DS, et al., 2019. 
Para conclusão, realizaram-se exames de finalização: radiografia panorâmica, telerradiografia lateral e radiografia periapical de canino superior direito. Verificou-se periodonto saudável, custo biológico aceitável na região periapical do dente 13 e ausência de danos aos dentes adjacentes (Figura 5).

Figura 5 - Finalização: radiografia periapical, região de canino superior direito.

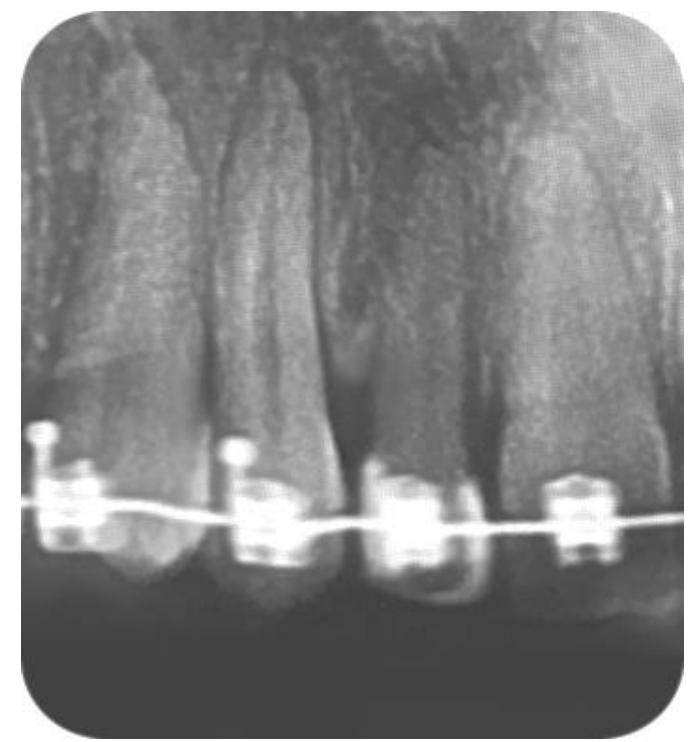

Fonte: Dias DS, et al., 2019.

Após remoção do aparelho ortodôntico fixo, foram instaladas as contenções: removível superior, placa de Hawley; e fixa inferior, 3x3. Registrou-se modelos de estudo, fotografias extrabucais e fotografias intrabucais finais (Figura 6).

Figura 6 - Fotografias intrabucais finais. A: frontal. B: lateral direita. C: lateral esquerda.
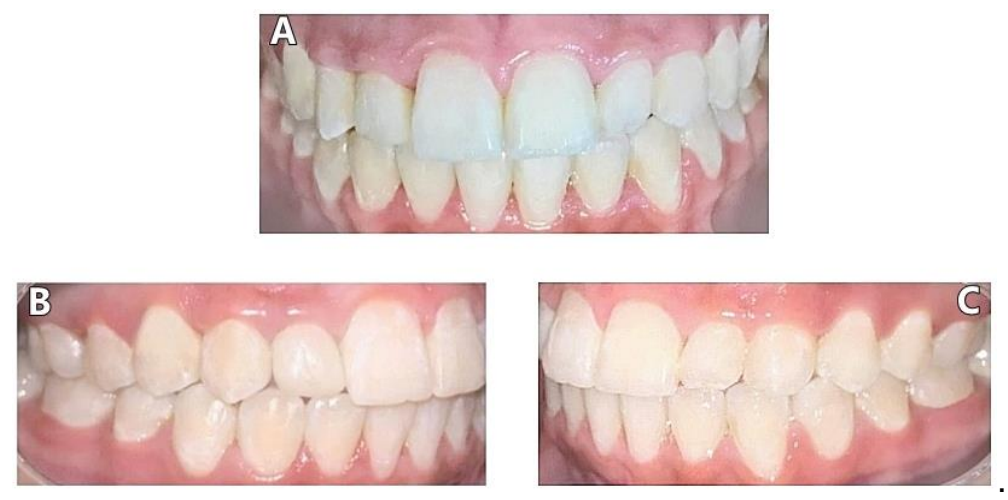

Legenda: A: frontal. B: lateral direita. C: lateral esquerda.

Fonte: Dias DS, et al., 2019

\section{DISCUSSÃO}

Estudos apontam que a impactação de caninos permanentes superiores está relacionada tanto a fatores gerais, quanto a fatores locais (GEBERT TJ, et al., 2014; NASCIMENTO LRXC, et al., 2016; SOARESSANTOS KS, et al., 2018, CRUVINEL MOB, et al., 2018). Dentre os principais fatores gerais estão: hereditariedade, endocrinopatias e síndromes com má formação craniofacial. Por sua vez, os fatores locais mais associados são: trajeto de erupção tortuoso, falta de espaço na arcada dentária, distúrbios na sequência de erupção dos dentes permanentes, traumatismo em dentição decídua, retenção prolongada ou 
perda prematura do canino decíduo, agenesia dos incisivos laterais permanentes, má posição do germe dentário, dilaceração radicular, anquilose dos caninos permanentes e presença de cistos, tumores ou supranumerários na região (ALVES EP, et al., 2014). Essa condição acomete o sexo feminino em uma proporção até $3: 1$, sendo dez vezes mais frequente na maxila do que na mandíbula, localizando na região palatina em $60-80 \%$ dos casos e podendo ser bilateral em $8 \%$ das ocorrências (MARCELINO VCS, et al., 2017).

Alves EP, et al. (2014) encontraram uma prevalência de 3,5\% de caninos superiores retidos ao avaliarem uma amostra de 372 radiografias panorâmicas. Nesse estudo a idade se mostrou um importante fator de influência, sendo a impactação mais prevalente nos pacientes de 13 a 30 anos.

De acordo com Carvalho AAB, et al. (2017), os exames radiográficos são fundamentais para determinar a posição e contexto espacial dos caninos inclusos. Com finalidade de diagnóstico, utilizam-se principalmente radiografias panorâmicas, periapicais, oclusais, telerradiografia lateral e tomografia computadorizada.

As técnicas radiográficas convencionais, sobretudo as radiografias panorâmicas, apresentam limitações para avaliação de caninos superiores não irrompidos. Exigem exames complementares como a radiografia oclusal de maxila ou periapicais pela técnica de Clark. No entanto, apesar de permitirem verificar se o canino está localizado na região vestibular ou palatina, não especificam a relação com os dentes adjacentes e possíveis perdas de estrutura radicular de incisivos laterais (CARDOSO MA, et al., 2011).

A tomografia computadorizada de feixe cônico mostra-se superior aos exames radiográficos convencionais, fornecendo imagens tridimensionais. Permite demonstrar precisamente a localização do canino incluso, estruturas adjacentes comprometidas e achados incidentais. Especialmente, possibilita revelar o grau de reabsorção de incisivos centrais e laterais (CARVALHO AAB, et al., 2017).

A utilização prévia do aparelho quadrihélice de Ricketts permite o restabelecimento da forma do arco superior, mostrando importante contribuição na correção das discrepâncias transversais e melhoria da fisiologia respiratória. Em adição, tal medida coopera para significante abreviação do tempo de nivelamento e alinhamento dentário (DUARTE MS, 2006).

Segundo Cardoso MA, et al. (2011), as principais medidas terapêuticas para caninos permanentes impactados são: exodontia do canino permanente e fechamento dos espaços com tratamento protético, transplante autógeno, exposição cirúrgica e tracionamento ortodôntico, ou ausência de tratamento imediato, exigindo acompanhamento em longo prazo. Para esses autores, o tracionamento parece ser a melhor modalidade de tratamento.

Em concordância, Cruvinel MOB, et al. (2018) recomendam o tracionamento e reservam a extração do canino permanente apenas aos casos de anquilose, reabsorção externa ou interna, raiz com grande dilaceração, severa impactação, risco de reabsorção radicular dos dentes adjacentes, alterações patológicas impeditivas, ou resistência do paciente ao tracionamento ortodôntico.

A exposição cirúrgica seguida por tracionamento utilizando cantilever representa uma estratégia de tratamento frequentemente utilizada para tratamento de caninos permanentes superiores inclusos. $O$ cantilever consiste em um braço de força, empregado na técnica do arco segmentado de Burstone. Essa técnica permite o máximo controle dos sistemas de forças, ao segmentar 0 arco em unidades ativas e passivas (CRUVINEL MOB, et al., 2018).

O tracionamento pode ser realizado principalmente através de três maneiras: técnica da laçada cervical, em desuso devido necessidade de extensa remoção óssea, risco reabsorção radicular externa e anquilose; técnica da perfuração da coroa, procedimento que apresenta dificuldade de acesso e riscos de danos pulpares; e técnica de colagem de acessório na coroa, menos invasiva, embora necessite de campo operatório seco para a colagem adequada (MARCELINO VCS, et al., 2017).

A técnica de colagem de acessório associada ao uso de cantilever permite a manutenção dos princípios biomecânicos para controle dos efeitos colaterais. Dessa maneira, fica assegurada uma sequência clínica simplificada, com minimização de desconforto ao paciente (CRUVINEL MOB, et al., 2018). 


\section{CONSIDERAÇÕES FINAIS}

O tratamento de canino permanente superior impactado apresentado no presente estudo permitiu uma abordagem conservadora e menos dispendiosa. A utilização da tomografia computadorizada, a terapêutica de expansão transversal da arcada superior com quadrihélice de Ricketts e o tracionamento ortodôntico pela técnica de colagem de acessório associada a cantilever mostraram resultados funcionais e estéticos positivos.

\section{AGRADECIMENTOS}

Agradecimentos a todos os docentes, discentes, servidores técnico-administrativos e pacientes do curso de especialização em Ortodontia da Associação Brasileira de Odontologia (ABO) em Montes Claros-MG que prestaram importantes contribuições a esta pesquisa.

\section{REFERÊNCIAS}

1. ALMEIDA RR, et al. Abordagem da impactação e/ou irrupção ectópica dos caninos permanentes: considerações gerais, diagnóstico e terapêutica. Revista Dental Press de Ortodontia e Ortopedia Facial, 2001; 6(1): 93-116.

2. ALVES EP, et al. Prevalência e posição de caninos superiores impactados e sua relação com reabsorção radicular. Revista da Faculdade de Odontologia da UPF, 2014; 19(2): 180-184.

3. ANGLE EH. Classification of malocclusion. Dental Cosmos, 1899; 41(3): 248-264.

4. BARIANI RC, et al. Orthodontic traction of impacted upper canines using the VISTA technique. Journal of Clinical Orthodontics, 2017; 51(2): 76-85.

5. BISHARA SE. Impacted maxillary canines: a review. American Journal of Orthodontics, 1992; 101(2): $159-171$.

6. CAPELOZZA FILHO L, et al. Perfuração do esmalte para o tracionamento de caninos: vantagens, desvantagens, descrição da técnica cirúrgica e biomecânica. Dental Press Journal of Orthodontics, 2011; 16(5): 172-205.

7. CAPELOZZA FILHO L. Diagnóstico em ortodontia. 2. ed. Maringá: Dental Press Editora, 2012; 81p.

8. CAPPELLETTE $M$, et al. Caninos permanentes retidos por palatino: diagnóstico e terapêutica: uma sugestão técnica de tratamento. Revista Dental Press de Ortodontia e Ortopedia Facial, 2008; 13(1): 60-73.

9. CARDOSO MA, et al. Tracionamento de canino permanente superior: relato de caso clínico. Revista Clínica de Ortodontia Dental Press, 2011; 10(4): 108-121.

10. CARVALHO AAB, et al. Importância da tomografia computadorizada de feixe cônico na avaliação de canino incluso na maxila. Revista Brasileira de Odontologia, 2017; 74(2): 143-149.

11. CRUVINEL MOB, et al. Utilização de cantilever para tracionamento de canino impactado. OrtodontiaSPO, 2018; 51(1): 74-79.

12. DAMANTE SC, et al. Tracionamento de caninos inclusos: diagnóstico e terapêutica. Archives of Health Investigation, 2017; 6(12): 580-585.

13. DUARTE MS. O aparelho quadrihélice (Quad-helix) e suas variações. Revista Dental Press de Ortodontia e Ortopedia Facial, 2006; 11(2): 128-156.

14. ERICSON S, KUROL PJ. Resorption of incisors after ectopic eruption of maxillary canines. A CT study. The Angle Orthodontist, 2000; 70(6): 415-423.

15. GEBERT TJ, et al. Dental transposition of canine and lateral incisor and impacted central incisor treatment: A case report. Dental Press Journal of Orthodontics, 2014; 19(1): 106-112.

16. MACENO IS, et al. Orthodontic traction technique: Clinical case report based on literature. Integrative Molecular Medicine, 2018; 5(1): 1-3.

17. MARCELINO VCS, et al. Tratamento cirúrgico-ortodôntico do dente 33: relato de caso clínico. Archives of Health Investigation, 2017; 6(7): 304-307.

18. NASCIMENTO LRXC, et al. Severa impactação de canino superior: relato de caso. Revista Odontológica de Araçatuba, 2016; 37(3): 15-19.

19. PRIMO BT, et al. Dentes retidos: novas perspectivas de localização. Revista da Faculdade de Odontologia da UPF, $2011 ; 16(1)$ : 95-99.

20. SILVA AC, et al. Root length and alveolar bone level of impacted canines and adjacent teeth after orthodontic traction: a long-term evaluation. Journal of Applied Oral Science, 2017; 25(1): 75-81.

21. SOARES-SANTOS KS, et al. Impactação bilateral de caninos superiores: relato de caso. Revista da Academia Brasileira de Odontologia, 2018; 7(1): 32-35.

22. STIVAROS N, MANDALL NA. Radiographic factors affecting the management of impacted upper permanent canines. Journal of Orthodontics, 2000; 27(2): 169-173. 